\title{
Uso prolongado de benzodiacepinas en el paciente anciano
}

\author{
Marcelo Domínguez-Cantero \\ FEA. Farmacia Hospitalaria. Servicio Andaluz de Salud (España)
}

\begin{abstract}
Las sociedades americanas y europeas de geriatría avalan la retirada de benzodiacepinas en pacientes ancianos tratados de forma prolongada, ya que su uso se asocia a trastornos del equilibrio, caídas, sedación y confusión. En nuestro estudio describimos el uso de benzodiacepinas en pacientes mayores de 65 años. Se seleccionó a la población atendida en un centro de salud urbano. Se definió como tratamiento prolongado aquellos con una duración superior a seis meses. Los datos se obtuvieron del sistema de información de tarjeta sanitaria facilitados por la comunidad autónoma. Se identificaron los pacientes mayores de 65 años tratados con benzodiacepinas a partir de los datos de facturación electrónica de las recetas de éstos fármacos dispensados en oficinas de farmacia durante el periodo enero-septiembre de 2016. De los 19399 pacientes atendidos en este centro, 6766 tenían una edad mayor de 65 años. El $92.8 \%$ de los pacientes con más de 65 años estaban tratados con benzodiazepinas. 1263 realizaban un uso prolongado de benzodiacepinas, $92.18 \%$ del total. La práctica habitual difiere de las recomendaciones sobre un uso limitado de benzodiacepinas de forma prolongada en pacientes ancianos. Lormetazepan, lorazepam y alprazolam son las benzodiacepinas más prescritas. Pueden priorizarse estas benzodiacepinas a la hora de iniciar programas de desprescripción de benzodiacepinas en la población y proponer otras alternativas no farmacológicas para tratar el insomnio en ancianos.
\end{abstract}

Palabras clave: Benzodiacepinas, ancianos, seguridad.

Prolonged use of benzodiazepines in the elderly patient. The American and European societies of geriatrics support the withdrawal of benzodiazepines in elderly patients treated for a prolonged period, since its use is associated with balance disorders, falls, sedation and confusion. In our study we describe the use of benzodiazepines in patients older than 65 years. The population attended in an urban health center was selected. Prolonged treatment was defined as those lasting longer than six months. The data was obtained from the health card information system provided by the autonomous community. We identified patients older than 65 years treated with benzodiazepines from the electronic billing data of the prescriptions of these drugs dispensed in pharmacy offices during the period January-September 2016. Of the 19399 patients treated in this center, 6766 were over 65 years of age. $92.8 \%$ of patients older than 65 years were treated with benzodiazepines. 1263 used long-term benzodiazepines, $92.18 \%$ of the total. The usual practice differs from the recommendations on a limited use of benzodiazepines for a long time in elderly patients. Lormetazepan, lorazepam and alprazolam are the most prescribed benzodiazepines. These benzodiazepines can be prioritized when initiating programs of benzodiazepine deprescription in the population and propose other nonpharmacological alternatives to treat insomnia in the elderly.

Keywords: Benzodiazepines, elderly, safety.

Correspondencia: Marcelo Domínguez-Cantero. Farmacia Hospitalaria. Servicio Andaluz de Salud. Paseo Capitán Conforto, s/n. C.P.: 11110. San Fernando, Cádiz (España). E-mail: $\underline{\text { marcelodcantero@gmail.com }}$ 
Las benzodiacepinas es grupo de psicofármacos con indicación principalmente en el uso a corto plazo del insomnio y trastornos de ansiedad, aunque son utilizados para tratar convulsiones en la epilepsia o contracturas musculares. Se caracterizan por un mecanismo de acción farmacológico a través de la unión al receptor del neurotransmisor ácido gamma amino butírico. Las benzodiacepinas se unen al receptor gabaérgico con mayor afinidad que el propio neurotransmisor. Como consecuencia de la unión se produce un aumento de la frecuencia de la apertura del canal de cloro y por lo tanto un incremento de la transmisión inhibitoria del neurotransmisor Ácido $\gamma$-aminobutírico (GABA). Se plantea que según el lugar de unión en los receptores GABA la acción de las benzodiacepinas es más ansiolítica o se potencia el efecto sedante. Las benzodiacepinas clásicas interactúan de forma aleatoria con los receptores de forma que es esperable una acción hipnótica y ansiolítica. Las nuevas generaciones de benzodiacepinas, como los denominados "grupo Z" (zolpidem, zopiclona) tienen un carácter más selectivo en su unión obteniendo un marcado efecto hipnótico (Catálogo de Medicamentos Consejo General de Colegios Oficiales Farmacéuticos, 2018). La clasificación de las benzodiacepinas según su vida media (Azparren y García, 2014) (ver Tabla 1).

Tabla 1. Clasificación de las benzodiacepinas según su vida media plasmática

\begin{tabular}{|c|c|c|}
\hline FARMACOCINÉTICA & PRINCIPIO ACTIVO & VIDA MEDIA (h) \\
\hline \multirow{7}{*}{$\begin{array}{l}\text { Acción corta (menos de } 8 \\
\text { horas) }\end{array}$} & Brotizolam & $3-8$ \\
\hline & Midazolam & $1-5$ \\
\hline & Triazolam & $3-5$ \\
\hline & Bentazepam & $2-5$ \\
\hline & Clotiazepam & $5.8-6.3$ \\
\hline & Zolpidem & $1.5-2.4$ \\
\hline & Zopiclona & 5 \\
\hline \multirow{8}{*}{ Acción media ( $8-24$ horas) } & Flunitrazepam & $15-30$ \\
\hline & Loprazolam & $4-15$ \\
\hline & Lormetazepam & $11-30$ \\
\hline & Alprazolam & $12-15$ \\
\hline & Bromazepam & $10-20$ \\
\hline & Clobazam & 18 \\
\hline & Ketazolam & $6-25$ \\
\hline & Lorazepam & $11-30$ \\
\hline \multirow{6}{*}{$\begin{array}{lccc}\text { Acción } & \text { larga (más } & \text { de } 24 \\
\text { horas) } & \text { Mezcla } & \text { insulina } \\
\text { humana } & & & \end{array}$} & Flurazepam & $24-100$ \\
\hline & Quazepam & $40-55$ \\
\hline & Clorazepato & $30-48$ \\
\hline & Clordiazepóxido & $1.5-4$ \\
\hline & Diazepam & $20-100$ \\
\hline & Halazepam & $30-100$ \\
\hline
\end{tabular}

El consumo de ansiolíticos en el territorio español es superior a la media europea según un informe publicado por la Agencia Española del Medicamento y Productos Sanitarios (AEMPS, 2014) se ha estimado que la prevalencia del consumo de hipnóticos sedantes en España durante el año 2011 fue del 11.4\%. Con un incremento cercano al 60\% durante el periodo 2000-2012. En función de la vida media de duración 
de acción, los ansiolíticos e hipnóticos más consumidos fueron los de acción intermedia, seguidos de los de acción larga. El mayor incremento de uso se registró también en los de acción intermedia, seguidos de los de acción corta 70.4\% y 44.1\% respectivamente.

El perfil de efectos adversos de las benzodiacepinas en la mayoría de los pacientes aceptable. Siendo mínima la aparición de reacciones adversas en tratamientos agudos. De forma general, las indicaciones sobre la duración adecuada que se recogen en la ficha técnica de las benzodiacepinas recomiendan utilizar (Ficha técnica diazepam, 2018):

- La dosis mínima eficaz hasta alcanzar un efecto clínico adecuado en los pacientes, debiendo ser la duración del tratamiento lo más corto posible.

- De forma general, el tratamiento no debe ser superior a un intervalo comprendido entre las ocho a 12 semanas, incluyendo la retirada gradual, necesaria para evitar efecto rebote y síndrome de abstinencia.

- Tratamientos superiores a seis meses de duración no mostraron beneficio clínico.

Por tanto, cuando se realizan tratamiento prolongados superiores a seis meses la eficacia no está demostrada y aumentan el riesgo de efectos adversos. En resumen, existe una relación beneficio-riesgo desfavorable cuando aumentamos el tiempo de tratamiento con este grupo de fármacos. Entre los efectos adversos más comunes que se han descrito se encuentran la sedación, debilidad muscular, ataxia, trastornos cognitivos y dependencia psíquica y fisca. No obstante, en determinados grupos de población y cuando se realizan tratamientos prolongados la tasa de eventos de efectos adversos aumenta. (Danza, Cristiani, y Tamosiunas, 2009).

En pacientes de edad avanzada se producen cambios en la farmacodinamia y farmacocinética que hacen que exista una mayor sensibilidad a nivel del sistema nervioso central al efecto de estos fármacos y acumulación plasmática de los mismos, especialmente en las benzodiacepinas de acción larga. (Mangoni y Jackson, 2003). Por lo tanto, en ancianos se recomienda de forma general usar la mitad de la dosis.

También se asocia el uso de benzodiacepinas con en pacientes de edad avanzada el doble de riesgo de fractura de cadera (Cumming y Le Conteur, 2003) incremento del grado de demencia o deterioro cognitivo (Madhusoodanan y Bogunovic, 2004) y aumento de la mortalidad entre los pacientes tratados con benzodiacepinas (Weich, Pearce, Croft, Singh, Crome, Bashford, y Frisher, 2014). En éste grupo de población existe cierta unificación de criterios en una prescripción seleccionada y de duración limitada de benzodiacepinas. De forma resumida, diversas sociedades científicas en el campo de la geriatría a nivel internacional recomiendan:

- La Sociedad Americana de Geriatría (Beers Criteria, 2015) indican evitar las benzodiacepinas de acción corta y media por un balance beneficio-riesgo negativo, sobre las de acción larga recomienda reservar su uso en determinadas indicaciones (vértigos 
severos, deshabituación alcohólica, medicación preanestésica, ansiedad grave generalizada).

- Los criterios españoles STOPP-START (Delgado, Muñoz, Montero, Sánchez, Gallagher, y Cruz-Jentoft, 2009) sobre el cese de medicación potencialmente inapropiada en paciente ancianos recomiendan la retirada de benzodiacepinas cuyo tratamiento sea superior a cuatro semanas. La sociedad europea no encuentra indicación de tratamientos con duración más prolongada. En contra de aumentar el tiempo de tratamiento riesgo de sedación prolongada, confusión, pérdida de equilibrio, caídas y accidentes de tráfico. La retirada deber realizarse de forma gradual, para evitar la aparición del síndrome de abstinencia.

- Dentro de la lista PRISCUS (Holt, Schmiedl, y Thürmann, 2010) de origen alemán desaconseja las benzodiacepinas de acción corta, éstos son preferibles a los de vida media larga. En todos los casos se recomienda iniciar con la dosis más baja, el menor tiempo posible, vigilar la función cognitiva y psiquiátrica.

En pacientes ancianos es recomendable valorar la necesidad de iniciar una benzodiacepina y evaluar la posibilidad de retirada una vez instaurado el tratamiento. En este contexto han surgido estrategias de deprescripción de benzodiacepinas. Consisten en la retirada gradual de benzodiacepinas en pacientes cuyo beneficio riesgo es negativo. No obstante las estrategias consisten la planificación de una retirada gradual de benzodiacepinas de duración prolongada, intervenciones psicológicas, fomentar unos buenos hábitos de higiene del sueño tales como: evitar alcohol o estimulantes, ir a la cama sólo cuando se tiene sueño, evitar las siestas, no tener en la habitación distracciones o ruidos (televisión, radio), practicar ejercicio antes de acostarse (Grupo de Trabajo de la Guía de Práctica Clínica para el Manejo de Pacientes con Insomnio en Atención Primaria, 2009). Estas estrategias varían en función de la organización sanitaria que las emita. Pueden tratarse de simple información al usuario sobre el problema a largo plazo del consumo de benzodiacepinas, aportando un plan de retirada gradual (Azparren y García, 2014) intercambiar la dosis de benzodiacepina actual a la dosis equivalente de diazepam.

Los estudios sobre deprescripción son heterogéneos y son necesarios diseños de ensayos clínicos para evidenciar la utilidad de un programa de deprescripción. Las últimas guías basadas en la evidencia que se han publicado al respecto indican: ofertar la retirada en todos los pacientes en tratamiento con benzodiacepinas por insomnio primario mayores de 65 años y en aquellos entre 18 y 64 años en tratamiento con benzodiacepinas durante más de cuatro semanas (Pottie, Thompson, Davies, Grenier, Sadowski, Welch et al., 2018).

El objetivo de nuestro estudio fue describir el uso de benzodiacepinas en pacientes mayores de 65 años. 


\section{MÉTODO}

\section{Participantes}

Para realizar el estudio se incluyó a la población atendida en un centro de salud de atención primaria urbano de la ciudad de Pamplona.

\section{Instrumentos}

Se seleccionaron los pacientes procedentes de una base de datos de la Comunidad Foral de Navarra que alberga datos de prescripción electrónica en atención primaria. Los datos estaban anonimizados.

\section{Procedimiento}

Se identificaron todos los pacientes mayores de 65 años tratados con benzodiacepinas a partir de los datos de facturación electrónica de las recetas de éstos fármacos dispensados en oficinas de farmacia. Se recogieron los datos durante el periodo enero a septiembre de 2016. Se seleccionaron variables demográficas (sexo, edad) principio activo del grupo de benzodiacepinas, número de principios activos por paciente en el último mes, principios activos prescritos. Se definió como tratamiento prolongado aquellos con una duración superior a seis meses.

\section{RESULTADOS}

De los 19.399 pacientes atendidos en este centro, 6.766 tenían una edad mayor de 65 años. Se seleccionaron en función del rango de edad establecidos a 1.370 pacientes $(20.25 \%)$ que se encontraban tratados con una o más benzodiacepinas. El $73.7 \%$ eran mujeres, promedio de 78.01 (65-103) años de edad.

Estratificando el uso de benzodiacepinas por rango de edad se obtuvo la siguiente distribución: entre 65 y 74 años 470 (34.31\%); entre 75 y 84 años 434 pacientes (31.67\%); entre 85 y 94 años 290 (21.17\%); mayor de 95 años 176 (12.85\%).

El $11.31 \%$ se encontraban en tratamiento con dos o más benzodiacepinas diferentes. $92.8 \%$ de los pacientes estaban tratados con siete benzodiacepinas.

La distribución en porcentaje de pacientes tratados con benzodiacepinas fue el siguiente: lormetazepam (30.77\%), lorazepam (22.77\%), alprazolam (13.21\%), zolpidem $(10.15 \%)$, bromazepam $(6.7 \%)$, diazepam $(4.81 \%)$, clorazepato potásico $(3.64 \%)$, loprazolam $(2.1 \%)$, ketazolam (1.3\%), flurazepam (1.2\%).

El resto de principios activos con menos del 1\%. 1263 realizaban, en el momento del estudio, un uso prolongado de tratamiento con benzodiacepinas, correspondiente al $92.18 \%$ del total. 


\section{DISCUSIÓN Y CONCLUSIONES}

Los datos sugieren que existe una práctica habitual de uso prolongado superior a seis meses en la mayoría de los pacientes tratados con benzodiacepinas. Estos datos no están en concordancia con las recomendaciones actuales de uso en pacientes de edad avanzada: tratamientos agudos y periodos cortos de duración. Parece que en nuestra muestra existe una sobreutilización de éstos psicofármacos. Podemos extrapolar que su uso se debe, como ocurre en la mayoría de los casos descritos a la indicación de insomnio o ansiolítico. No obstante, no se recogió como una de las variables del estudio la indicación para la que se prescribió en cada paciente. Los datos de prevalencia obtenidos son superiores, aproximadamente en el doble de pacientes en tratamiento con benzodiacepinas cuando se compara con el estudio de utilización de ansiolíticos e hipnóticos durante los años 2000-2012 publicado por la Agencia Española del Medicamento, con una diferencia de 11.1 vs 20\% (AEMPS, 2013). En dicho estudio no se limita la edad en el dato de prevalencia contrariamente al límite inferior de 65 años que se estableció como criterio de inclusión.

Tampoco se indica si el mayor consumo, además de ser debido a un mayor porcentaje de población tratada puede ser debida a una duración excesiva de los tratamientos. Mediante las medidas de dosis habitante día (DHD) no es posible discernir entre una mayor población o tratamientos prolongados. Por otra parte, esta diferencia puede ser debida al tamaño muestral inferior en nuestro estudio.

En nuestro estudio puede existir un importante sesgo de selección a la hora de determinar tratamientos de duración prolongada. El motivo de seleccionar duración prolongada como aquellos con tratamiento mayor de seis meses como criterio de inclusión surge al ceñirse a un documento oficial como es la ficha técnica del medicamento. En éstos se informa que tratamientos superiores a seis meses no mostraron beneficio clínico. En contraposición, la opinión del panel de expertos de sociedad europea de geriatría recomienda la retirada gradual de benzodiacepinas en aquellos tratamientos superiores a cuatro semanas. Sin embargo, la duración del tratamiento no parece poseer la misma importancia para las distintas sociedades de geriatría. Las recomendaciones de las Sociedad Americana de Geriatría (Beers Criteria, 2015) no indica duración máxima recomendada de tratamiento. Probablemente, la elección como criterio de inclusión de una duración menor podría aumentar el número de pacientes incluidos en el estudio y la modificación de los resultados finales.

Lormetazepan, lorazepam y alprazolam son las benzodiacepinas más prescritas. Todas ellas benzodiacepinas de acción intermedia. Dicho patrón de consumo coincide con lo descrito en el informe sobre el consumo de ansiolíticos e hipnóticos elaborado por la Agencia Española del Medicamento y Productos Sanitarios en 2013, tanto en la agrupación por semivida plasmática (siendo los más consumidos los de vida 
intermedia) como en los principios activos (más consumidos fueron alprazolam y lorazepam). Por otra parte, señalar que esta familia de benzodiacepinas no es la recomendada en pacientes ancianos, en los que se deben priorizar el uso de principios activos de acción corta. Además, es reseñable una baja tasa de pacientes tratados con diazepam, benzodiacepina de elección en pautas de retirada, puede sugerir un escaso número de pacientes con tratamiento en retirada. Los datos no son comparables con los aportados por la Agencia Española del Medicamento ya que el consumo de éste informe se aporta en dosis por a habitante y día al año y nuestros datos corresponden a tasas respecto al total de pacientes tratados en un periodo inferior al año. También es destacable entre los resultados aquellos pacientes en tratamiento con dos o más benzodiacepinas, con porcentaje cercano al diez por ciento. Según indica la ficha técnica de este grupo de fármacos se destina su uso a la monoterapia, no está recomendada la combinación de fármacos que tienen idéntica diana farmacológica.

El manejo de benzodiacepinas combinadas suele ser complicado debido a la dependencia de los pacientes. Una duplicidad de benzodiacepinas puede provocar un aumento en el riesgo de caída, deterioro cognitivo del paciente e incluso en incremento de la mortalidad. No obstante, desconocemos la indicación los pacientes con duplicidad de éste grupo terapéutico y si existe justificación en su uso. Hubiera sido conveniente analizar la historia clínica en estos casos para comprender el motivo de la prescripción. Diversos estudios relacionaron el uso de benzodiacepinas con una mayor mortalidad, incremento del riesgo de fractura de fémur, deterioro cognitivo y otras reacciones adversas.

Una limitación de nuestro estudio es su diseño descriptivo. Debido a esto, no podemos relacionar con una mayor tasa de eventos adversos en aquellos pacientes con mayor duración de tratamiento. Sería de interés en futuros estudios o para ésta misma cohorte realizar un seguimiento sobre la seguridad de las benzodiacepinas o si su uso precipitó la prescripción en cascada de otros fármacos para paliar efectos adversos propios de la benzodiacepinas.

A la vista de los resultados sería oportuno confirmar si se mantiene un incremento de la prevalencia en otros centros de salud, sin rangos de edad y la temporalidad de la misma. De esta forma podríamos valorar como acción prioritaria dentro de las políticas sanitarias la implantación de estrategias de deprescripción de benzodiacepinas. Podrían priorizarse las benzodiacepinas de nuestro estudio (lormetazepam, lorazepam, y alprazolam) a la hora de iniciar programas de desprescripción de benzodiacepinas en la población que incluyan como primera línea de tratamiento, en el caso del uso de benzodiacepinas como hipnóticos, son medidas de higiene del sueño y proponer otras alternativas no farmacológicas para tratar el insomnio en ancianos. De forma general podemos concluir que la prescripción de benzodiacepinas es inadecuada en la mayoría de los pacientes tratados. 
La causa principal es el uso prolongado más allá de los seis meses de tratamiento. La práctica habitual reflejada en nuestro estudio difiere de las recomendaciones propuestas por sociedades científicas en el campo de la geriatría sobre un uso limitado de benzodiacepinas en pacientes ancianos. A pesar de esto, debido al diseño descriptivo del estudio no podemos relacionar una duración prolongada con una mayor tasa de eventos adversos relacionados con la medicación.

\section{REFERENCIAS}

Agencia Española del Medicamento y Productos Sanitarios (2013). Utilización de medicamentos ansiolíticos e hipnóticos en España durante el período 2000-2012. Disponible en: www.aemps.es

American Geriatrics Society (2015). Beers Criteria Update Expert Panel. American Geriatrics Society. Updated Beers Criteria for Potentially Inappropriate Medication. Use in Older Adults. Journal of the American Geriatrics Society, 63, 2227-2246.

Azparren, A., y García, I. (2014). Estrategias para la deprescripción de benzodiazepinas. Boletín de Información Farmacoterapéutica de Navarra, 22(2), 1-12.

Catálogo de Medicamentos Consejo General de Colegios Oficiales Farmacéuticos (2018). Base de datos Bot PLUS 2.0 del Consejo General de Colegios Oficiales de Farmacéuticos.

Cumming, R.G., y Le Conteur, D.G. (2003). Benzodiazepines and Risk of Hip Fractures in Older People. CNS Drugs, 17(11), 825-837.

Danza, A., Cristiani, F., y Tamosiunas, G. (2009). Riesgos asociados al uso de benzodiazepinas. Archivos de Medicina Interna, 4,103-107.

Delgado, E., Muñoz, M., Montero, B., Sánchez, C., Gallagher, P.F., y Cruz-Jentoft, A.J. (2009). Inappropriate prescription in older patients: the STOPP/START criteria. Revista Española de Geriatría y Gerontología, 44(5), 273-279.

Ficha técnica diazepam (2018). Base de datos Bot PLUS 2.0 del Consejo General de Colegios Oficiales de Farmacéuticos.

Guía de Práctica Clínica para el Manejo de Pacientes con Insomnio en Atención Primaria (2009). Plan de Calidad para el Sistema Nacional de Salud del Ministerio de Sanidad y Política Social. Unidad de Evaluación de Tecnologías Sanitarias. Comunidad de Madrid. Disponible en: http://www.guiasalud.es/GPC/GPC_465_Insomnio_Lain_Entr_compl.pdf

Holt, S., Schmiedl, S., y Thürmann, P.A. (2010). Potentially Inappropriate Medications in the Elderly: The PRISCUS List. Deutsches Ärzteblatt International,107, 543-551.

Madhusoodanan, S., y Bogunovic, O.J. (2004). Safety of benzodiazepines in the geriatric population. Expert Opinion Drug Safety, 3(5), 485-493.

Mangoni, A.A., y Jackson, S.H.D. (2003). Age-related changes in pharmacokinetics and pharmacodynamics: basic principles and practical applications. British Journal of Clinical Pharmacology, 57(1), 6-14.

Pollmann, A.S., Murphy, A.L., Bergman J.C., y Gardner D.M. (2015). Deprescribing benzodiazepines and Z-drugs in community-dwelling adults: a scoping review. BMC Pharmacology and Toxicology, 4(16), 19.

Pottie, K., Thompson, W., Davies, S., Grenier, J., Sadowski, C.A., Welch, V., Farrell, B. (2018). Deprescribing benzodiazepine receptor agonists: Evidence-based clinical practice guideline. Canadian Family Physician, 64(5), 339-351. 
Weich, S., Pearce, H.L., Croft, P., Singh, S., Crome, I., Bashford, J., y Frisher, M. (2014). Effect of anxiolytic and hypnotic drug prescriptions on mortality hazards: retrospective cohort study. British Medical Journal, 19(348), 1-12.

Recibido: 10 de junio de 2018 Recepción Modificaciones: 15 de julio de 2018

Aceptado: 17 de julio de 2018 